\title{
Adaptive Symbol-Rate Free-Space-Optical Communications
}

\author{
William G. Cowley and Khoa D. Nguyen \\ ITR, University of South Australia, Mawson Lakes, South Australia \\ Dirk Giggenbach \\ German Aerospace Center (DLR), Wessling, Germany
}

\begin{abstract}
Free space optical communications through fading atmospheric channels can achieve improved throughput by using adaptive transmission techniques. This paper considers the use of adaptive symbol-rate and transmit-power methods. We use an idealised model of channel coding that assumes zero errors can be achieved above some SNR threshold and adjust the transmission rate and power as the channel varies to operate above the required threshold. Results are presented for two noise models appropriate to non-coherent photodetection. Substantial performance gains are demonstrated compared to non-adaptive transmission. Most of the benefit from adaptive power control can be obtained with modest peak to average power constraints.
\end{abstract}

\section{INTRODUCTION}

Free space optical (FSO) communications offers the potential for Gbit point-to-point wireless transmission without using any radio frequency spectrum. Unfortunately, fading caused by small variations in the atmosphere's refractive index, has limited the uptake of FSO communications (e.g. [1], [2]). During the last decade advances in channel coding have been applied to FSO links and demonstrated greatly improved bit error performance (e.g. [3], [4], [5]). While other approaches such as multiple transmitters, adaptive optics or the use of hybrid FSO and RF channels can further mitigate the effects of atmospheric scintillation, the use of powerful channel coding techniques is particularly attractive due to the low additional cost and large performance gains.

Given the relatively large FSO channel coherence time of milliseconds, interleaving over tens of milliseconds plus iterative channel coding methods can be used to achieve reliable transmission at a fixed information rate (e.g. [6]). A relatively small number of authors have explored adaptive methods that aim to vary the transmission rate to suit the channel conditions, either on a short term or long term basis. For example, in terrestrial applications as the channel attenuation varies due to cloud or rain attenuation, provided a feedback channel exists from the receiver to the transmitter, the encoder may adjust its code rate, say by puncturing, to suit slowly varying channel conditions (e.g. [7]). In satellite scenarios, [8] demonstrates adaptive datarates can provide gains up to a factor 3 in overall throughput for low-earth-orbit optical downlinks.

If the data rate is large enough and the feedback delay is small, adaptive methods may be applied on short time scales to reduce (or even stop) data transmission during FSO channel fades. This approach is similar to the adaptive methods used in RF communications, such as [9]. In [10], [11] the use of varying modulation and/or transmit power for FSO links is shown to provide useful increases in the average Mutual Information. The first of these papers and [12] also illustrate that a feedback path is not necessarily required for adaptive FSO methods as the fading in each direction of axially-aligned bidirectional FSO link may be highly correlated.

In this paper we continue the investigation into short-term adaptive FSO methods. We consider a link that can vary the symbol period $T$ over some small finite set of values, and possibly the transmit power, depending of channel state. We assume an ideal decoder that corrects all errors given that the received signal-to-noise ratio is above some given threshold and determine the throughput gain of the adaptive scheme compared to the use of a fixed symbol period. Since noise models for FSO links can vary significantly, we employ two different models and demonstrate the relative benefit in each case.

The rest of the paper is organised as follows. In Section II we introduce the system model including two options for noise. We include measured results showing a good match to these noise models. In Section III a method of channel coding, either for an adaptive or a fixed symbol-period scheme is described, together with simulations of the performance gain with adaptive selection of transmission rate, for various fading channels. In Section IV the adaptive scheme is extended to optimise optical transmit power over the same range of transmission rate options. The last section provides brief conclusions.

\section{SySTEM MODEL}

Figure 1 shows a model of a FSO link with adaptive symbol rate. We assume intensity modulation with direct detection. Coded symbols are represented by optical pulses of length $T$ seconds with mean transmit power $P$. The multiplier $h$ represents a lognormal fading channel, with power scintillation index $\sigma_{I}^{2}=E\left\{h^{2}\right\} /(E\{h\})^{2}-1$, normalised so that $E\{h\}=$ 1. Lognormal fading with varying scintillation levels has been used in this paper as provides a good match for measured FSO channels under a wide range of conditions (e.g. [1], [12]). We 


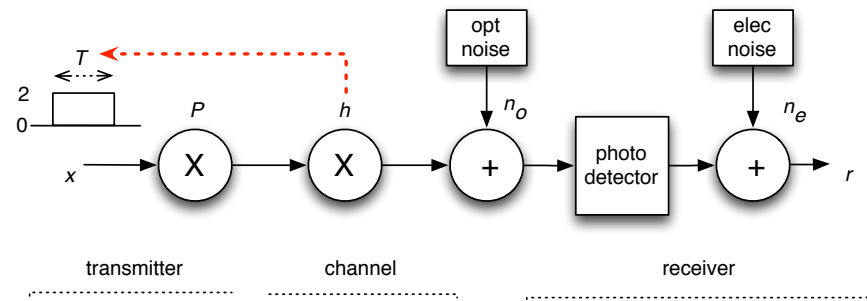

Fig. 1. FSO transmission model with adaptive symbol period controlled by channel state, including both optical and electrical noise components.

assume initially that both transmitter and receiver know the value of $h$ at all times and that the transmitter periodically adjusts the symbol period $T$, as described below, to optimise throughput. We assume an ideal conversion from received optical irradiance to electrical voltage with unit gain.

At the receiver the electrical signal after the photodetector can be written as

$$
r=x P h+n_{o}+n_{e}
$$

where $x$ is the transmitted symbol value and the last two terms represent optical and electrical noise sources.

A wide range of noise sources can affect FSO communications and a variety of models have been applied at both device and system levels (e.g. [13], [14]. In this paper we consider two simple noise models at either end of the normally assumed models: that the optical noise sources are small compared to electrical noise and the overall distribution is Gaussian, or that electrical noise can be neglected and Poisson statistics may be employed with the number of photons large enough to allow a Gaussian approximation. While (1) is a continuous-time model and $r$ would normally be the input to a matched filter, in the latter noise model it will be more convenient to consider the receiver front-end as a photon counting device.

\section{A. Noise Models}

1) Model 1: In this model the overall noise from both optical and electrical components is modelled as an additive white Gaussian noise (AWGN) term of constant power spectral density. We assume the received irradiance is converted to voltage with unit gain. Due to the unipolar optical signal and assuming on-off keying (OOK), the DC component of the noisy electrical signal can be neglected, which is equivalent to using symbol values of \pm 1 with the same optical power $P$. A matched filter and sampler is then used to provide an optimum detection statistic $z$ to a channel decoder.

Since the noise power within the receiver bandwidth is $N_{0} / 2 T$, by scaling the time units we may write the instantaneous signal to noise ratio at the receiver input as ${ }^{1}$

$$
\gamma_{e}=h^{2} P^{2} T
$$

\footnotetext{
${ }^{1}$ Note that the average SNR depends on the scintillation index since in this model $E\left\{h^{2}\right\}=1+\sigma_{I}^{2}$. To avoid later confusion we measure codec performance under different fading conditions via optical power $P$.
}

The model also applies to binary pulse position modulation (BPPM) although the normalisation factor will be different. Note in the above equation, unlike RF systems, the SNR depends on the square of the transmitted power but since the noise power is still proportional to the symbol rate, the SNR also depends on the symbol period.

2) Model 2: In contrast to the previous model dominated by electrical noise, in this case we neglect electrical noise and assume Poisson statistics for each received optical pulse. Given that the standard deviation for a Poisson random variable is the square root of the mean value, the SNR will be $(h P T)^{2} / h P T$. This is a common assumption for photon counting and ideal optical detectors, e.g. [13], but is difficult to achieve in practice as electrical noise is always present to some extent. Since $P T$ is proportional to the number of photons per bit (ppb), in this model performance will depend only on this quantity, rather than bit rate.

With respect to the modulations considered in this paper, this is a good model for the BPPM case where the same mean number of photons will be received for either symbol value. Assuming Gaussian statistics is reasonable if the number of photons in large enough. For the OOK case the assumption of signal independent Gaussian noise (SIGN) is clearly loose since in the absence of background radiation no photons will be received for the "off" symbol value. Nevertheless averaging signal and noise terms across the two symbols values still gives this expression, thus we have retained the same model:

$$
\gamma_{o}=h P T
$$

3) Comparison of noise models with sample APD results: Figure 2 illustrates the measured BER performance of a photoreceiver as bit rate and bandwidth was varied. These OOK measurements were made by the German Aerospace Center (DLR) with an InGaAs avalanche photodiode (APD) at $1550 \mathrm{~nm}$ followed by a fixed transimpedance amplifier and a variable-bandwidth amplifier [15]. Raw bit error rate was measured as the data rate, bandwidth and optical power was varied, while keeping the receiver performance close to optimum in terms of APD-voltage. This figure shows a summary of performance in terms of photons per bit to achieve the specified BERs.

Note that our second noise model predicts a constant number of $\mathrm{ppb}$ to achieve a given BER which would result in horizontal lines in Figure 2. Also shown in the figure are lines of ppb proportional to $\sqrt{(} T)$ which implies $P T \propto \sqrt{T}$ or constant $P^{2} T$ for a given BER, as predicted by our receiver model 1. The closer agreement of these measured results to the first model may be related to the method of only varying the receiver bandwidth via a separate filter-amplifier, while the TIA feedback impedance was kept constant in the equipment. However, with the measurements having a flatter slope towards lower data rates the receiver proves to be not completely thermal noise-limited. Therefore, to model practical APDreceivers, the exponent of $T$ in (2) has to be chosen somewhere between 1 and 2 . The degradation at the highest data rates 


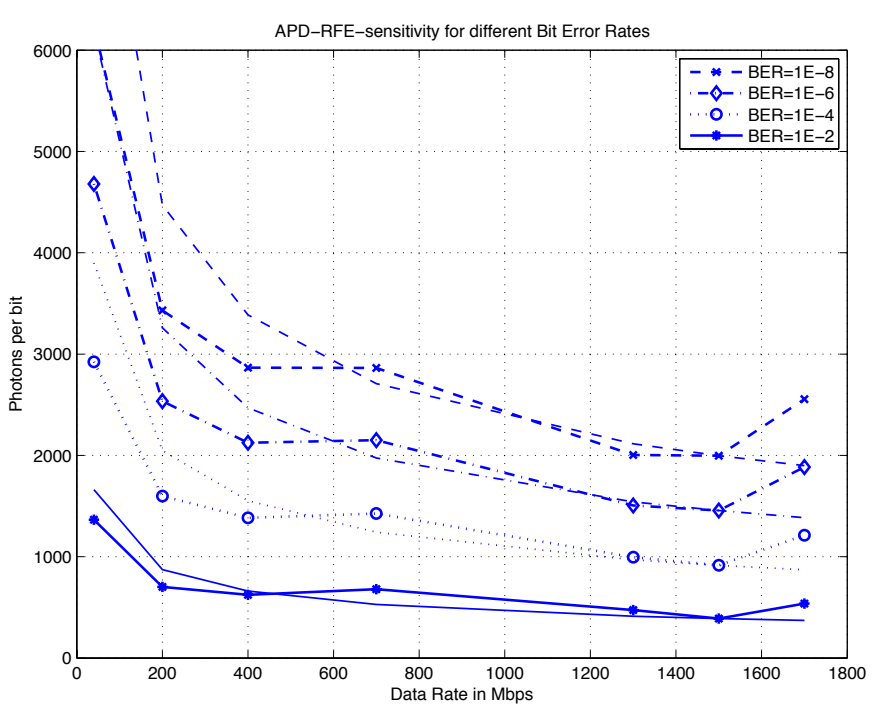

Fig. 2. Sample measurements of APD-receiver performance in photons per bit over a wide range of bit rates.

(1.7Gbps) in Figure 2 are due to bandwidth-limiting effects, while the receiver performs best at $1.5 \mathrm{Gbps}$.

\section{B. Adaptive Model}

In order to compare adaptive and fixed transmission-rate schemes for FSO links we will assume that each system is used in conjunction with the same powerful channel code. The fixed rate scheme is assumed to use a large interleaver, say 100 milliseconds, so that each codeword experiences the full range of channel fading. In the following the coded fixedrate throughput will be compared to the adaptive approach.

In contrast to the fixed scheme, we consider an adaptive scheme without interleaver where the symbol period is adjusted using (2) or (3) to achieve the required SNR for the decoder to converge with no errors. In a high-rate FSO link, even relatively long codewords of several thousand symbols can be transmitted over an interval during which the channel can be assumed constant amplitude (say 1 millisecond). In practice the choice of $T$ will be constrained to a finite set of values $\mathcal{T}=\left\{T_{1}, \ldots, T_{m}\right\}$, rather than continuously variable. Without loss of generality, we assume $T_{1} \geq T_{2} \geq \ldots \geq T_{m}$. We have simulated this situation in Section III.

Furthermore, we will consider jointly adapting symbol duration $T$ and transmit power $P$, subject to the peak power constraint $P \leq P_{\text {peak }}$ and average power constraint $\mathbb{E}\{P\} \leq P_{\text {av }}$. The adaptation rule is developed in Section IV, which brings significant gains in throughput performance.

Figure 3 shows sample BER performance for a rate 0.8 LDPC code versus optical power $P$. The dashed lines, from left to right, represent fading channels with $\sigma_{I}^{2}=0.5,1$ and 2 respectively. Since the error rate falls very rapidly with increasing transmit power in the "waterfall" region of the BER curve, we make the simple assumption that the BER drops to zero abruptly at a given threshold value of power $P$. This example is a regular LDPC code with variable nodes of

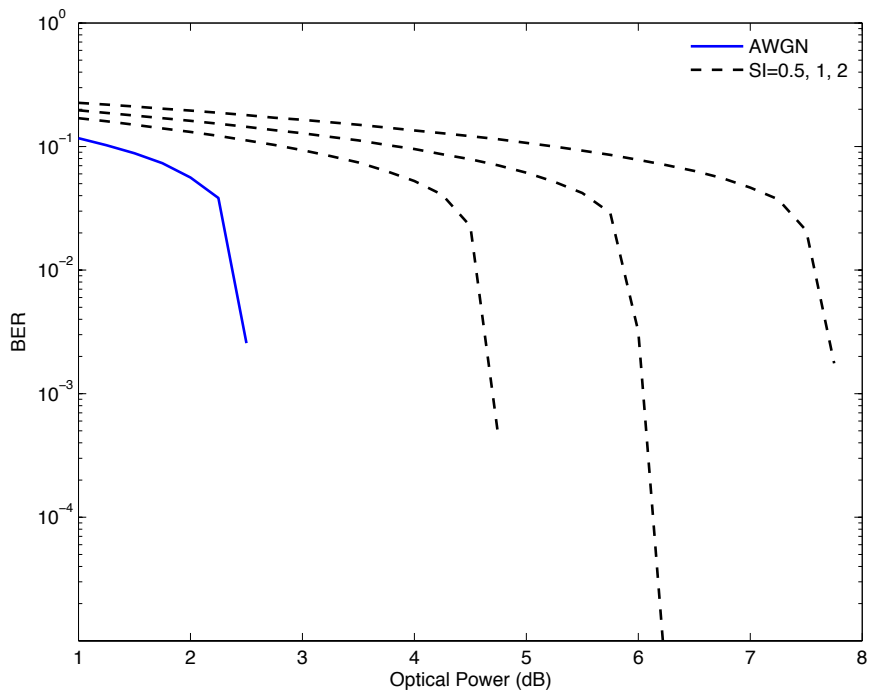

Fig. 3. Example of bit error rate performance for $N=10000, K=0.8$ LDPC code under AWGN and three LN fading channels, simulated with $z=$ $\pm h P+n$ where $n \sim N(0,1), T=1$.

degree 3. Optimised irregular codes would show even sharper threshold performance.

\section{AdAPTIVE TRANSMISSION}

Given the models outlined above the scheme has been simulated for the specific code mentioned in Figure 3. A BER threshold of $10^{-3}$ has been used to approximate the waterfall cliff. For example, in the non-fading case the optical power $P_{a}$ of $2.6 \mathrm{~dB}$ is assumed to give zero errors while $6.1 \mathrm{~dB}$ is required for the fading optical power threshold $P_{f}$ when the scintillation index is 1 .

Under these assumptions the first adaptive scheme has been simulated by generating a large number of fading channel samples and selecting the symbol period to keep $\gamma_{e}$ at least equal to the SNR required for the non-fading (AWGN) LDPC decoder, $P_{a}^{2}$. Keeping the optical power at $P_{f}$ for a fair comparison with the non-adaptive scheme, for each value of $h$, the minimum value of $T$ is selected from a discrete set $^{2}$ to ensure

$$
h^{2} P_{f}^{2} T \geq P_{a}^{2}
$$

The second model is the same except that now the symbol period is selected to ensure that the optical energy per pulse is maintained at the required non-fading decoder threshold. Using the same options for $T$ selection, for each $h$ we choose

$$
h P_{f} T \geq P_{a}
$$

In each case the relative throughput $R_{T P n}$ of the adaptive scheme to the non-adaptive scheme is the mean value of $T^{-1}$. Sample results for this code are given in Table I with the third and fourth columns for models 1 and 2 respectively.

This waterfall cliff approach has the advantage of giving a simple ratio in total throughput whereas more accurate

\footnotetext{
${ }^{2}$ Octave steps from $2^{-5}$ to 2
} 
TABLE I

SAMPLE THROUGHPUT RESULTS FOR THE ADAPTIVE TRANSMISSION MODELS AND CODE SHOWN IN FIGURE 3.

$\begin{array}{cccc}\sigma_{I}^{2} & P_{f} & R_{T P 1} & R_{T P 2} \\ 0.5 & 4.7 \mathrm{~dB} & 2.7 & 1.2 \\ 1 & 6.1 \mathrm{~dB} & 5.2 & 1.6 \\ 2 & 7.8 \mathrm{~dB} & 8.1 & 2.3\end{array}$

models of the decoder BER will not allow this. However the differences due to codec performance are still likely to be small compared to the variation due to the noise model employed.

\section{Power And Symbol Rate Adaptation}

In this section, we will jointly adapt the symbol duration $T$ and transmit power $P$ to improve the relative throughput achieved in Section III. For a system with a peak power constraint $P_{\text {peak }}$ and an average power constraint $P_{\text {av }}$, the optimal adaptive strategy solves the following optimisation problem

$$
\begin{cases}\text { Maximise }_{T(h), P(h)} & \int_{0}^{\infty} \frac{\mathbf{1}\left\{P(h)^{n} h^{n} T(h) \geq P_{a}^{n}\right\}}{T(h)} f_{h}(h) d h \\ \text { Subject to } & \int_{0}^{\infty} P(h) f_{h}(h) d h \leq P_{\mathrm{av}} \\ & P(h) \leq P_{\text {peak }} \\ & T(h) \in \mathcal{T},\end{cases}
$$

where $n=2$ and 1 for the first and second noise model correspondingly.

To solve the optimisation problem (6), we first have the following Propositions.

Proposition 1. Let $P^{*}(h), T^{*}(h)$ be the solution of (6), then

$$
P^{*}(h)= \begin{cases}\frac{P_{a}}{\left(T^{*}(h)\right)^{1 / n}} \frac{1}{h}, & h \in \mathcal{H} \\ 0, & \text { otherwise }\end{cases}
$$

for some $\mathcal{H} \subset \mathbb{R}$.

Sketch of Proof: The result can be obtained by a contradiction proof. Intuitively, the optimal scheme allocate just sufficient power to satisfy the non-fading LDPC decoding threshold $P_{a}^{n}$. However, if maintaining $P_{a}^{n}$ is too costly, for e.g. when $h$ is small, the transmitter saves power by turning off transmission.

Therefore the optimisation problem in (6) reduces to

$$
\begin{cases}\text { Maximise }_{T(h)} & \int_{0}^{\infty} \frac{1}{T(h)} f_{h}(h) d h \\ \text { Subject to } & \int_{0}^{\infty} \frac{P_{a}}{h T(h)^{1 / n}} f_{h}(h) d h \leq P_{\mathrm{av}} \\ & T(h) \geq \frac{P_{a}}{P_{\text {peak }}^{n} h^{n}} \\ & T(h) \in \mathcal{T} \cup\{\infty\},\end{cases}
$$

where we have replaced $P(h)$ by (7) and the element $\infty$ was included to cover the case $P(h)=0$, so that $T(h)=\infty, h \notin$ $\mathcal{H}$. The problem can be further simplified by the following Proposition.
Proposition 2. Assume that $T^{*}(h)$ is a solution of (8), then $T^{*}(h)$ is a non-increasing function of $h$.

Sketch of Proof: The proof is based on contradiction. Specifically, for a given adaptation rule $T(h)$, if there exists $h_{1} \geq h_{2}$ such that $T_{i}=T\left(h_{1}\right) \geq T\left(h_{2}\right)=T_{j}$, we can find a new adaptation rule $T^{\prime}(h)$ that outperforms $T(h)$ by interchanging $T\left(h_{1}\right)$ and $T\left(h_{2}\right)$.

Therefore, letting $T_{0}=\infty$, the optimal adaptation rule satisfies

$$
T(h)=T_{i}, h \in\left[h_{i}, h_{i+1}\right)
$$

for $i=0, \ldots, m$, where $h_{0}=0, h_{m+1}=\infty$ and $h_{1}, \ldots h_{m}$ is the solution of

$$
\begin{cases}\text { Maximise } & \sum_{i=1}^{m} \frac{1}{T_{i}} \int_{h_{i}}^{h_{i+1}} f_{h}(h) d h \\ \text { Subject to } & \sum_{i=1}^{m} \frac{1}{T_{i}^{1 / n}} \int_{h_{i}}^{h_{i}+1} \frac{1}{h} f_{h}(h) d h \leq \frac{P_{\text {av }}}{P_{a}} \\ & h_{i} \geq \frac{P_{a}}{P_{\text {peak }}} \frac{1}{T_{i}^{1 / n}}, i=1, \ldots, m .\end{cases}
$$

The optimisation problem is separable, and thus can be solved using the branch-and-bound linear programming technique.

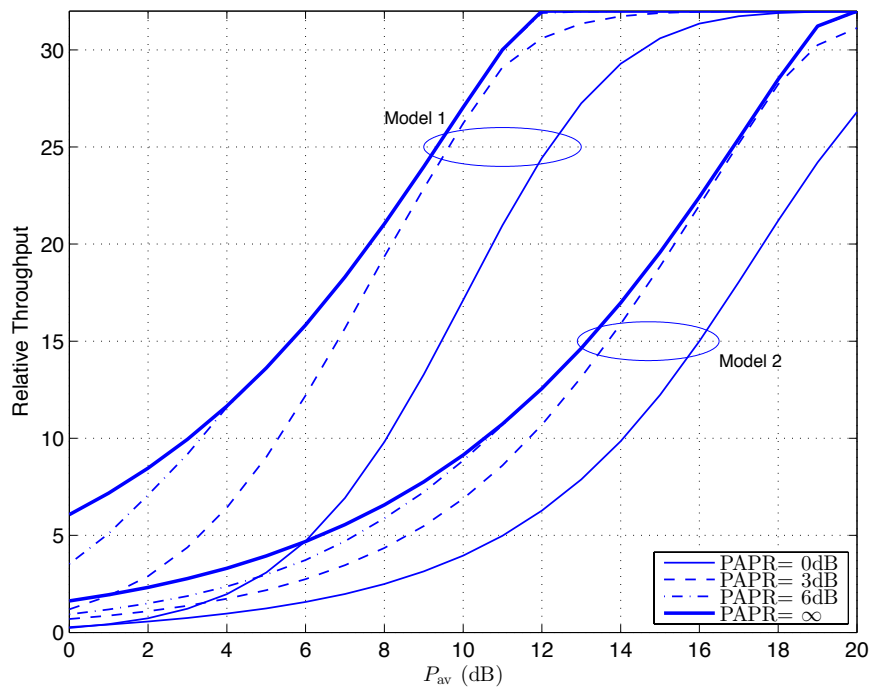

Fig. 4. Throughput obtained by power and symbol rate adaptation in channels with $\sigma_{I}^{2}=0.5$

We perform the optimisation for the available symbol rates $\mathcal{T}$ as in Section III with average power constraint $P_{\mathrm{av}}$ and peak power constraint $P_{\text {peak }}=\mathrm{PAPR} \times P_{\mathrm{av}}$. In this context, $\mathrm{PAPR}=0 \mathrm{~dB}$ corresponds to a non-power-adaptive system discussed in Section III; while PAPR $=\infty$ corresponds to a system without peak power constraint.

The throughputs achieved by power and symbol rate adaptation are represented in Figures 4, 5 and 6 for log-normal fading channels with scintillation indices $\sigma_{I}^{2}=0.5,1$ and 2 correspondingly. The figures show that significant gains in throughput can be achieved by power adaptation. Specifically, in systems without peak power constraint, power adaptation can bring more than $3 \mathrm{~dB}$ gains on average power in most cases. More importantly, most of these gains can be obtained 


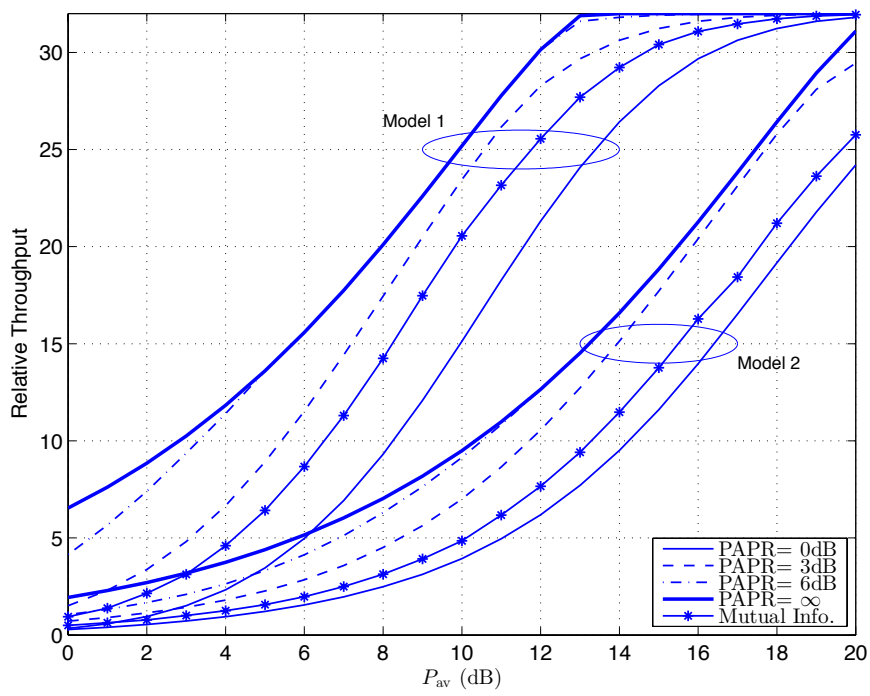

Fig. 5. Throughput obtained by power and symbol rate adaptation in channels with $\sigma_{I}^{2}=1.0$

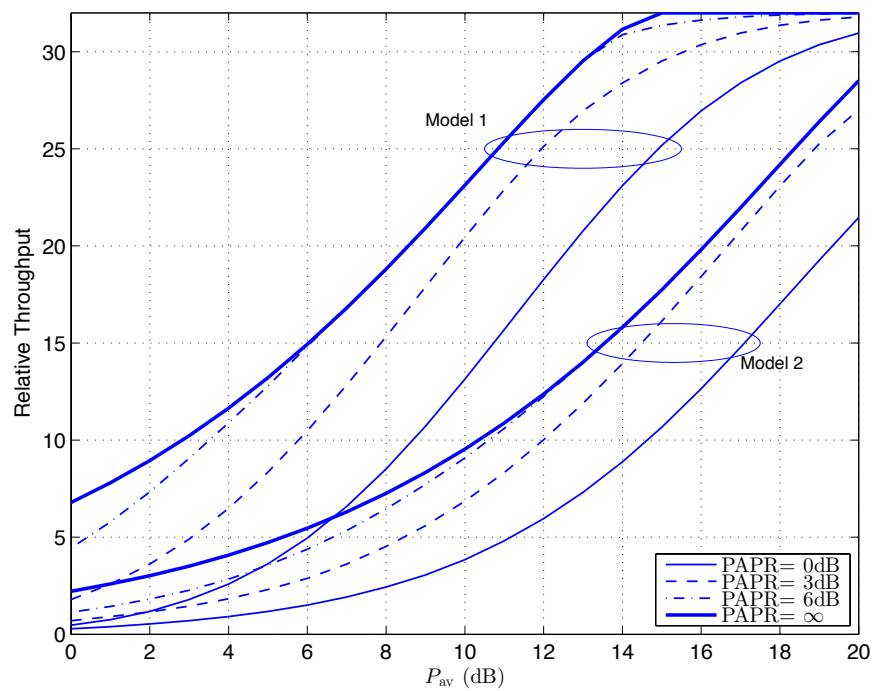

Fig. 6. Throughput obtained by power and symbol rate adaptation in channels with $\sigma_{I}^{2}=2.0$

by a PAPR as low as $6 \mathrm{~dB}$. At PAPR $=3 \mathrm{~dB}$, more than $2 \mathrm{~dB}$ gains on average power are observed.

Figure 5 also illustrates the mutual information $\bar{I}\left(P_{\mathrm{av}}\right)$ achieved by the transmission scheme with no power adaptation $(\mathrm{PAPR}=0)$ :

$$
\bar{I}\left(P_{\text {av }}\right) \triangleq \mathbb{E}_{h}\left\{\max _{T \in \mathcal{T}} \frac{I\left(P_{\mathrm{av}}^{n} h^{n} T\right)}{T}\right\},
$$

where $I(\rho)$ is the input-output mutual information of an optical channel with binary input, Gaussian noise and signal-to-noise ratio $\rho$. This simulation also used a $\mathrm{LN}$ fading channel with $\sigma_{I}^{2}=1$. It is observed that the code performance when there is no power adaptation is $2 \mathrm{~dB}(1 \mathrm{~dB})$ below the mutual information limit for model 1 (and 2 correspondingly). The performance loss is partly due to the suboptimality of the
LDPC code being used; and partly due to the omission of bad channel realisations in the adaptation approach. However, with 3dB PAPR, the power adaptive system can achieve better throughput compared to the non-power-adaptive mutual information limit, even with suboptimal LDPC codes.

\section{Conclusions}

This paper has described an adaptive transmission scheme suited to very high speed free-space-optical communication links. By assuming the transmitter has perfect knowledge of the current fading channel amplitude and can adjust its transmission rate in order to ensure convergence of a powerful high-rate channel code, we demonstrate by simulation that substantial improvements in average information bit rate are possible. This approach requires rapid adaption, say on millisecond timescales, but this is quite feasible for Gbit links. The performance benefit improves as the power scintillation increases.

Given the wide range of noise models commonly used for FSO communications, this paper illustrates the benefits of adaptive transmission for two different noise models. These are shown to have a large impact on the relative performance and clearly warrants further investigation including the use of signal dependent noise models where required. The paper assumes a modest set of symbol rate options, in octave steps. These values have not been optimised.

In addition to adaptive symbol-rate transmission at fixed optical power, we show that further improvements are possible with adaptive control of both symbol rate and transmit power. By considering the impact of the peak to average power ratio, we demonstrate that most of the gain due to adaptive power control can be realised with quite modest values of peak to average power. Finally, as a check on the ideal channel code assumption, the adaptive symbol-rate performance has been measured using a mutual information approach and shown to provide reasonably consistent estimates.

\section{REFERENCES}

[1] L. Andrews and R. Phillips, Laser beam propagation through random media, vol. 152. Society of Photo Optical, 2005.

[2] H. Willebrand and B. Ghuman, Free space optics: enabling optical connectivity in today's networks. Sams Publishing, 2002.

[3] D. Caplan, "Laser communication transmitter and receiver design," Journal of Optical and Fiber Communications Research, vol. 4, no. 4, pp. 225-362, 2007.

[4] N. Letzepis, I. Holland, and W. Cowley, "The gaussian free space optical mimo channel with q-ary pulse position modulation," Wireless Communications, IEEE Transactions on, vol. 7, no. 5, pp. 1744-1753, 2008.

[5] G. Karagiannidis, S. Arnon, J. Barry, R. Schober, and M. Uysal, "Guest editorial: optical wireless communications," Selected Areas in Communications, IEEE Journal on, vol. 27, pp. 1521 -1525, december 2009.

[6] W. Cowley, G. Lechner, C. Biggs, and K. Grant, "Transceiver design for reliable free space optical communications," in Communications Theory Workshop (AusCTW), 2011 Australian, pp. 94-99, IEEE, 2011.

[7] A. Eslami, S. Vangala, and H. Pishro-Nik, "Hybrid channel codes for efficient fso/rf communication systems," Communications, IEEE Transactions on, vol. 58, no. 10, pp. 2926-2938, 2010.

[8] N. Perlot and T. de Cola, "Throughput maximization of optical leoground links," in Proceedings of SPIE, vol. 8246, p. 82460V, 2012. 
[9] A. Goldsmith and S. Chua, "Adaptive coded modulation for fading channels," Communications, IEEE Transactions on, vol. 46, no. 5 , pp. 595-602, 1998.

[10] A. Khatoon, W. Cowley, and N. Letzepis, "Capacity of adaptive freespace optical channel using bidirectional links," in in Laser Communication and Propagation through the Atmosphere and Oceans Conference, SPIE,Optical Eng and Applications, August 2012.

[11] I. Djordjevic, "Adaptive modulation and coding for free-space optical channels," Optical Communications and Networking, IEEE/OSA Journal of, vol. 2, no. 5, pp. 221-229, 2010.
[12] D. Giggenbach, W. Cowley, K. Grant, and N. Perlot, "Experimental verification of the limits of optical channel intensity reciprocity," Applied Optics, vol. 51, no. 16, pp. 3145-3152, 2012.

[13] B. Oliver, "Thermal and quantum noise," Proceedings of the IEEE, vol. 53, no. 5, pp. 436-454, 1965.

[14] R. Gagliardi and S. Karp, Optical communications. New York, WileyInterscience, 445 p., 1976.

[15] D. Giggenbach, "Practical receiver models with variable bandwidth for free-space optical communications.," in preparation, 2012. 